\title{
Single-vendor multi-buyer vendor managed inventory with discrete demand and stockout
}

\author{
Mehdi Seifbarghy ${ }^{1}$ and Ali Pourebrahim Gilkalayeh ${ }^{2}$
}

${ }^{1}$ Department of Industrial Engineering, Alzahra University, Tehran, Iran

${ }^{2}$ Faculty of Industrial and Mechanical Engineering, Qazvin Branch, Islamic Azad University, Qazvin, Iran

\begin{abstract}
This paper deals with the operational issues of a two-echelon single-vendor multibuyer supply chain model under vendor managed inventory (VMI) mode of operation. Considering multiple buyers and assuming unsatisfied demands to be backordered at the buyers are major extensions in this paper. The vendor replenishes a single product to the buyers. The operational parameters of buyers are replenishment frequency, ordering cycle time, replenishment quantity and maximum number of authorised stockout while for the vendor, we consider ordering cycle time and order quantity as the operational parameters. Considering replenishment frequency of each buyer as the major decision variable of the model, the total inventory system cost is stated in terms of the replenishment frequency and other operational parameters are obtained as given equations. Since the objective function of the given model is nonlinear involving several integer variables, two metaheuristic-based algorithms [i.e., genetic algorithm (GA) and simulated annealing (SA)] are utilised in order to solve the problem; the results indicate that the first algorithm gives better solutions compared with the second.
\end{abstract}

Keywords: supply chain management, vendor managed inventory, VMI, discrete demand, stockout, simulated annealing, genetic algorithm. 